\title{
Some Fifth Degree Integration Formulas for Symmetric Regions
}

\author{
By A. H. Stroud
}

1. Introduction. Here we discuss some approximate integration formulas of the form

$$
\int \underset{s_{n}}{\ldots} \int f\left(x_{1}, \cdots, x_{n}\right) d x_{1} \cdots d x_{n} \cong \sum_{i=1}^{N} A_{i} f\left(\nu_{i 1}, \cdots, \nu_{i n}\right) .
$$

Here $S_{n}$ is a region in $n$-dimensional Euclidean space $(n \geqq 2)$, the $A_{i}$ are constants and the $\left(\nu_{i 1}, \cdots, \nu_{i n}\right)$ are points in the space. We discuss a general class of 5 th degree formulas for a class of regions $S_{n}$ which includes the $n$-dimensional cube and sphere; a precise description of the regions to which our discussion applies will be given in the next section. Each formula in the class contains no more than $N=2^{n}(n+1)$ points and has all positive coefficients $A_{i}$. For the $n$-sphere we give constants in four useful formulas which contain $2^{n}(n+1), 2^{n} n+1,2^{n+1}-1$ and $2^{n}+2 n$ points. The $2^{n}(n+1)$ point formula has all coefficients equal. The $2^{n}+2 n$ point formula has fewer points than any other known 5 th degree formula in which all coefficients are positive (for the $n$-sphere, $n \geqq 4$ ). The corresponding formulas for the $n$-cube are not as useful since, for most values of $n$, they have some points which lie outside the cube.

Previously, Hammer and Stroud [3] have given formulas of 5th degree for the $n$-cube and $n$-sphere which use only $2 n^{2}+1$ points. Those formulas, however, have the undesirable feature that, for the $n$-sphere $(n \geqq 5)$, some of the coefficients $A_{i}$ are negative. The only other known general class of 5 th degree formulas for the $n$-sphere, for arbitrary $n$, are the spherical product formulas; these contain $3^{n}-3^{n-1}+1$ points and have all positive coefficients. (Spherical product formulas were first described, for $n=2,3$, by Peirce [6,7], and for arbitrary $n$ independently by Hetherington [4] and Mysovskih [5]; an alternative description is given by Stroud and Secrest [8].) For the 3-sphere Ditkin [1] gives a 5th degree formula containing 13 points and positive coefficients.

2. A General Class of Formulas. To construct a 5th degree formula let us take the following points and coefficients

$$
\left( \pm \nu_{i 1}, \pm \nu_{i 2}, \cdots, \pm \nu_{i n}\right) A_{i}, \quad i=1, \cdots, n+1
$$

where the $\nu_{i k}$ and $A_{i}$ are parameters to be determined. Here we mean that all of the (at most $2^{n}$ ) points

$$
\left( \pm \nu_{j 1}, \pm \nu_{j 2}, \cdots, \pm \nu_{j n}\right)
$$

are taken with the same coefficient $A_{j}, j=1, \cdots, n+1$. If certain of the $\nu_{j k}$ are zero then the number of points with coefficient $A_{j}$ is less than $2^{n}$.

The regions $S_{n}$ to which our discussion will apply can be described precisely in

Received April 20, 1965. 
terms of the monomial integrals

$$
I\left(x_{1}^{\alpha_{1}} \cdots x_{n}^{\alpha_{n}}\right) \equiv \int \underset{s_{n}}{\ldots} \int x_{1}^{\alpha_{1}} x_{2}^{\alpha_{2}} \cdots x_{n}^{\alpha_{n}} d x_{1} \cdots d x_{n}
$$

(where the $\alpha_{k}$ are all nonnegative integers). We assume $S_{n}$ has the property that for $0 \leqq \alpha_{1}+\cdots+\alpha_{n} \leqq 5$ the only nonzero monomial integrals are

$$
I(1), I\left(x_{i}{ }^{2}\right), I\left(x_{i}{ }^{2} x_{j}{ }^{2}\right) \quad i, j=1, \cdots, n,
$$

and that

$$
\begin{gathered}
I\left(x_{1}{ }^{2}\right)=\cdots=I\left({x_{n}}^{2}\right), \\
I\left(x_{1}{ }^{4}\right)=\cdots=I\left({x_{n}}^{4}\right), \\
I\left(x_{1}{ }^{2} x_{2}{ }^{2}\right)=\cdots=I\left(x_{n-1}^{2} x_{n}{ }^{2}\right) .
\end{gathered}
$$

Requiring that (1) be exact for the monomials (3) means that a certain system of $(n+1)(n+2) / 2$ equations must be satisfied. This system can be written in the matrix form

$$
\mathbf{X}^{T} \mathbf{A X}=\mathbf{C}
$$

where $\mathbf{X}^{T}$ is the transpose of $\mathbf{X}$,

$$
\begin{aligned}
& \mathbf{X}=\left[\begin{array}{ccccc}
\nu_{11}^{2} & \nu_{12}^{2} & \cdots & \nu_{1 n}^{2} & 1 \\
\nu_{21}^{2} & \nu_{22}^{2} & \cdots & \nu_{2 n}^{2} & 1 \\
\vdots & \vdots & \cdots & \vdots & \vdots \\
\nu_{n+1,1}^{2} & \nu_{n+1,2}^{2} & \cdots & \nu_{n+1, n}^{2} & 1
\end{array}\right] \\
& \mathbf{A}=2^{n}\left[\begin{array}{ccccc}
A_{1} & 0 & \cdots & 0 & 0 \\
0 & A_{2} & \cdots & 0 & 0 \\
\vdots & \vdots & \cdots & \vdots & \vdots \\
0 & 0 & \cdots & A_{n} & 0 \\
0 & 0 & \cdots & 0 & A_{n+1}
\end{array}\right] \text {, } \\
& \mathbf{C}=\left[\begin{array}{ccccc}
c_{40} & c_{22} & \cdots & c_{22} & c_{20} \\
c_{22} & c_{40} & \cdots & c_{22} & c_{20} \\
\vdots & \vdots & \cdots & \vdots & \vdots \\
c_{22} & c_{22} & \cdots & c_{40} & c_{20} \\
c_{20} & c_{20} & \cdots & c_{20} & c_{00}
\end{array}\right] \\
& c_{\alpha \beta}=\int \underset{s_{n}}{\ldots} \int x_{i}{ }^{\alpha} x_{j}^{\beta} d x_{1} \cdots d x_{n}, \quad i, j=1, \cdots, n \text {. }
\end{aligned}
$$

(Here we have assumed all the $\nu_{i j} \neq 0$. If, for a certain $i, k$ of the $\nu_{i 1}, \cdots, \nu_{i n}$ are zero then the $i$ th diagonal element of the matrix $\mathbf{A}$ must be $2^{n-k} A_{i}$.)

It is not difficult to see that matrix $C$ is positive definite. Equation (4) states that matrices $\mathbf{A}$ and $\mathbf{C}$ are congruent. Thus if we can find parameters $\nu_{i j}, A_{i}$ which satisfy (4) with the $\nu_{i j}^{2}$ all real then, by the law of inertia for quadratic forms (Gantmaher [2]), it must turn out that the $A_{i}$ are all positive. 
3. Formula I: $2^{n}(n+1)$ Points. For the points (2) let us choose the following

$$
\begin{aligned}
& ( \pm \eta, \pm \eta, \pm \eta, \cdots, \pm \eta, \pm \eta) \\
& ( \pm \nu, \pm \lambda, \pm \lambda, \cdots, \pm \lambda, \pm \lambda) \\
& ( \pm \lambda, \pm \nu, \pm \lambda, \cdots, \pm \lambda, \pm \lambda) \\
& ( \pm \lambda, \pm \lambda, \pm \lambda, \cdots, \pm \lambda, \pm v)
\end{aligned}
$$

all with the same coefficient $A$. Then the system (4) reduces to

$$
\begin{aligned}
2^{n}(n+1) A & =c_{00} \\
\nu^{2}+(n-1) \lambda^{2}+\eta^{2} & =\frac{(n+1) c_{20}}{c_{00}} \\
\nu^{4}+(n-1) \lambda^{4}+\eta^{4} & =\frac{(n+1) c_{40}}{c_{00}} \\
2 \nu^{2} \lambda^{2}+(n-2) \lambda^{4}+\eta^{4} & =\frac{(n+1) c_{22}}{c_{00}}
\end{aligned}
$$

It is not too difficult to show that the solution of this system can be written as

$$
\begin{gathered}
\eta^{2}=\frac{c_{20}}{c_{00}} \pm \sqrt{ }\left(\frac{c_{40}}{c_{00}}+\frac{(n-1) c_{22}}{c_{00}}-n\left(\frac{c_{20}}{c_{00}}\right)^{2}\right) \\
n \lambda^{4}-2\left[\frac{(n+1) c_{20}}{c_{00}}-\eta^{2}\right] \lambda^{2}+\frac{(n+1) c_{22}}{c_{00}}-\eta^{4}=0, \\
\nu^{2}=\frac{(n+1) c_{20}}{c_{00}}-\eta^{2}-(n-1) \lambda^{2} .
\end{gathered}
$$

For the $n$-sphere $x_{1}{ }^{2}+x_{2}{ }^{2}+\cdots+x_{n}{ }^{2} \leqq 1$

$$
\begin{aligned}
c_{00} & =\frac{\pi^{n / 2}}{\Gamma\left(\frac{n}{2}+1\right)}, & c_{20} & =\frac{1}{n+2} c_{00}, \\
c_{40} & =\frac{3}{(n+2)(n+4)} c_{00}, & c_{22} & =\frac{1}{(n+2)(n+4)} c_{00}
\end{aligned}
$$

and there are four solutions to (5). Choosing the negative sign in the expression for $\eta^{2}$ and the smallest of the two corresponding solutions for $\lambda^{2}$ gives the solution

$$
\begin{aligned}
\eta^{2} & =\frac{(n+4)-2 \sqrt{ }(n+4)}{(n+2)(n+4)} \\
\lambda^{2} & =\frac{n(n+4)+2 \sqrt{ }(n+4)-\sqrt{ }(2(n+1)(n+2)(n+4))}{n(n+2)(n+4)} \\
\nu^{2} & =\frac{n(n+4)+2 \sqrt{ }(n+4)+(n-1) \sqrt{ }(2(n+1)(n+2)(n+4))}{n(n+2)(n+4)}
\end{aligned}
$$

for which all the points in the formula lie inside the $n$-sphere. In fact, the points $( \pm \eta, \cdots, \pm \eta)$ lie on a sphere of radius 


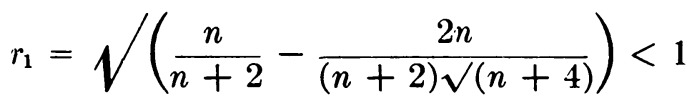

and the remaining points lie on a sphere of radius

$$
r_{2}=\sqrt{\left(\frac{n}{n+2}+\frac{2}{(n+2) \sqrt{ }(n+4)}\right)<1 .}
$$

The other three possible solutions to (5) always give some points which lie outside the sphere for $n>2$ (or are complex).

For the $n$-cube, with vertices $( \pm 1, \pm 1, \cdots, \pm 1)$, the solution for which all points are inside the cube for the most values of $n(2 \leqq n \leqq 6)$ is:

$$
\begin{aligned}
& \eta^{2}=\frac{5+2 \sqrt{ } 5}{15} \\
& \lambda^{2}=\frac{5 n-2 \sqrt{ } 5-2 \sqrt{ }(5(n+1))}{15 n}, \\
& \nu^{2}=\frac{5 n-2 \sqrt{ } 5+2(n-1) \sqrt{ }(5(n+1))}{15 n} .
\end{aligned}
$$

4. Formula II: $2^{n} n+1$ Points. For the points and coefficients (2) we choose

$$
\begin{aligned}
& \left(\begin{array}{llllll}
0, & 0, & 0, & \cdots & 0, & 0
\end{array}\right) \quad A_{0} \\
& ( \pm \nu, \pm \lambda, \pm \lambda, \cdots, \pm \lambda, \pm \lambda) \quad A_{1} \\
& ( \pm \lambda, \pm \nu, \pm \lambda, \cdots, \pm \lambda, \pm \lambda) \quad A_{1} \\
& ( \pm \lambda, \pm \lambda, \pm \dot{\lambda}, \cdots, \pm \lambda, \pm v) \quad \dot{A}_{1}
\end{aligned}
$$

System (4) becomes

$$
\begin{aligned}
A_{0}+2^{n} n A_{1} & =c_{00}, \\
2^{n} A_{1}\left[\nu^{2}+(n-1) \lambda^{2}\right] & =c_{20}, \\
2^{n} A_{1}\left[\nu^{4}+(n-1) \lambda^{4}\right] & =c_{40}, \\
2^{n} A_{1}\left[2 \nu^{2} \lambda^{2}+(n-2) \lambda^{4}\right] & =c_{22}
\end{aligned}
$$

with solution$$
\text { (n) }
$$$$
2^{n} A_{1}
$$$$
\frac{c_{20}^{2}}{c_{40}+(n-1) c_{22}}, \quad A_{0}=c_{00}-\frac{n c_{20}^{2}}{c_{40}+(n-1) c_{22}},
$$$$
\nu^{2}=\frac{c_{40}+(n-1) c_{22} \pm(n-1) \sqrt{ }\left(\left\{c_{40}-c_{22}\right\}\left\{c_{40}+(n-1) c_{22}\right\}\right)}{n c_{20}}
$$$$
\lambda^{2}=\frac{c_{40}+(n-1) c_{22} \mp \sqrt{ }\left(\left\{c_{40}-c_{22}\right\}\left\{c_{40}+(n-1) c_{22}\right\}\right)}{n c_{20}} .
$$

For the $n$-sphere, choosing the positive sign in $\nu^{2}$, gives the solution

$$
\begin{aligned}
2^{n} A_{1} & =\frac{(n+4)}{(n+2)^{2}} c_{00}, \quad A_{0}=\frac{4}{(n+2)^{2}} c_{00}, \\
\nu^{2} & =\frac{n+2+(n-1) \sqrt{ }(2(n+2))}{n(n+4)}, \quad \lambda^{2}=\frac{n+2-\sqrt{ }(2(n+2))}{n(n+4)}
\end{aligned}
$$


with all points inside the sphere for all $n$. In fact all points except the origin lie on a sphere of radius

$$
r=\sqrt{\left(\frac{n+2}{n+4}\right) .}
$$

For the $n$-cube the solution (6) which gives all points inside the cube for the most values of $n(2 \leqq n \leqq 5)$ is

$$
\begin{aligned}
& 2^{n} A_{1}=\frac{5}{5 n+4} c_{00}, \quad A_{0}=\frac{4}{5 n+4} c_{00}, \\
& \nu^{2}=\frac{5 n+4+2(n-1) \sqrt{ }(5 n+4)}{15 n}, \quad \lambda^{2}=\frac{5 n+4-2 \sqrt{ }(5 n+4)}{15 n} .
\end{aligned}
$$

5. Formula III: $2^{n+1}-1$ Points. For the points and coefficients (2) we choose

$$
\begin{aligned}
& \left( \pm \nu_{1}, \pm \lambda, \pm \lambda, \cdots, \pm \lambda, \pm \lambda\right) \quad A_{1} \\
& \left(0, \quad \pm \nu_{2}, \pm \lambda, \cdots, \pm \lambda, \pm \lambda\right) \quad A_{2} \\
& \begin{array}{ccccccc}
\vdots & \vdots & \vdots & \cdots & \vdots & \vdots & \vdots \\
(0, & 0, & 0, & \cdots & 0, & \left. \pm \nu_{n}\right) & A_{n}
\end{array} \\
& (0, \quad 0,0, \cdots, 0,0) \quad A_{n+1} \text {. }
\end{aligned}
$$

Equations (4) are

$$
\begin{aligned}
2^{n} A_{1}+2^{n-1} A_{2}+\cdots+2 A_{n}+A_{n+1} & =c_{00} \\
{\left[2^{n} A_{1}+\cdots+2^{n-i+2} A_{i-1}\right] \lambda^{2}+2^{n-i+1} A_{i} \nu_{i}{ }^{2} } & =c_{20} \\
{\left[2^{n} A_{1}+\cdots+2^{n-i+2} A_{i-1}\right] \lambda^{4}+2^{n-i+1} A_{i} \nu_{i}{ }^{4} } & =c_{40} \\
{\left[2^{n} A_{1}+\cdots+2^{n-i+2} A_{i-1}\right] \lambda^{4}+2^{n-i+1} A_{1} \nu_{i}{ }^{2} \lambda^{2} } & =c_{22}
\end{aligned} \quad i=1,2, \cdots, n .
$$

Equations (7) have the unique solution

$$
\begin{aligned}
\lambda^{2} & =\frac{c_{22}}{c_{20}}, \quad \nu_{i}{ }^{2}=\frac{c_{40}+(i-1) c_{22}}{c_{20}}, \quad i=1, \cdots, n, \\
2^{n-i+1} A_{i} & =\frac{c_{20}^{2}\left[c_{40}-c_{22}\right]}{\left[c_{40}+(i-2) c_{22}\right]\left[c_{40}+(i-1) c_{22}\right]}, \quad i=2, \cdots, n \\
2^{n} A_{1} & =\frac{c_{20}^{2}}{c_{40}}, \quad A_{n+1}=c_{00}-\frac{n c_{20}^{2}}{c_{40}+(n-1) c_{22}} .
\end{aligned}
$$

For the $n$-sphere we have

$$
\begin{aligned}
\lambda^{2} & =\frac{1}{n+4}, \quad \nu_{i}{ }^{2}=\frac{i+2}{n+4}, \quad i=1, \cdots, n, \\
2^{n-i+1} A_{i} & =\frac{2(n+4)}{(i+1)(i+2)(n+2)} c_{00}, \quad i=1, \cdots, n, \\
A_{n+1} & =\left[\frac{2}{n+2}\right]^{2} c_{00}
\end{aligned}
$$


and all points except the center lie on a sphere of radius

$$
r=\sqrt{ }\left(\frac{n+2}{n+4}\right) \text {. }
$$

For the $n$-cube some points lie outside the cube for $n \geqq 3$.

6. Formula IV: $2^{n}+2 n$ Points. For the points and coefficients (2) we choose

$$
\begin{aligned}
& ( \pm \eta, \pm \eta, \cdots, \pm \eta, \pm \eta) \quad A_{1} \\
& ( \pm \nu, 0, \cdots, 0, \quad 0) \quad A_{2} \\
& \begin{array}{cccccc}
\vdots & \vdots & \cdots & \vdots & \vdots & \vdots \\
(\mathbf{0}, & 0, & \cdots & 0, & \pm v) & A_{2} .
\end{array}
\end{aligned}
$$

System (4) is

$$
\begin{aligned}
2^{n} A_{1}+2 n A_{2} & =c_{00} \\
2^{n} A_{1} \nu^{2}+2 A_{2} \eta^{2} & =c_{20}, \\
2^{n} A_{1} \nu^{4}+2 A_{2} \eta^{4} & =c_{40} \\
2^{n} A_{1} \eta^{4} & =c_{22} .
\end{aligned}
$$

The values of $\nu^{2}, \eta^{2}$ satisfy

$$
\begin{aligned}
\left(c_{20}^{2}-c_{00} c_{22}\right) \nu^{4}-2 c_{20}\left(c_{40}-c_{22}\right) \nu^{2}+\left(c_{40}-c_{22}\right)^{2}+n c_{22}\left(c_{40}-c_{22}\right) & =0, \\
{\left[n c_{20}^{2}-c_{00}\left(c_{40}-c_{22}\right)\right] \eta^{4}-2 n c_{20} c_{22} \eta^{2}+n c_{22}^{2}+c_{22}\left(c_{40}-c_{22}\right) } & =0,
\end{aligned}
$$

where corresponding to the largest value of $\nu^{2}$ we must use the smallest value of $\eta^{2}$ and vice versa.

For the $n$-sphere the solution

$$
\begin{aligned}
\nu^{2} & =\frac{n+4-\sqrt{ }(2(n+4))}{n+4}, & \eta^{2} & =\frac{n(n+4)+2 \sqrt{ }(2(n+4))}{\left(n^{2}+2 n-4\right)(n+4)}, \\
2^{n} A_{1} & =\frac{1}{(n+2)(n+4) \eta^{4}} c_{00}, & 2 A_{2} & =\frac{2}{(n+2)(n+4) \nu^{4}} c_{00}
\end{aligned}
$$

gives all points inside the $n$-sphere for $n \geqq 4$.

For the $n$-cube, $n \geqq 2$, there is no solution for which all the points are inside.

7. Concluding Remarks and Examples. For the $n$-sphere the above $2^{n+1}-1$ point formula can be constructed in another way which is rather interesting.

As mentioned above, the spherical product of $n$, 3-point one-dimensional formulas contains $3^{n}-3^{n-1}+1$ points. A 5 th degree spherical product formula can be constructed, however, using fewer points than this. Let us take, as in the usual spherical product, the 3-point one-dimensional Gauss formula for the radial component. For each of the $n-1$ angular components let us take 5th degree, 4-point one-dimensional Lobatto formulas (which include the end points among the four points). By a somewhat tedious counting argument, which we will not carry out here, it can be shown that the resulting formula contains only $2^{n+1}-1$ distinct 
TABLE 1. Approximate values of integrals

\begin{tabular}{l|c|c}
\hline Number of points & $J_{4}$ & $J_{8}$ \\
\hline $2 n^{2}+1$ & 3.4854745 & 2.6958484 \\
$2^{n}+2 n$ & 3.4767683 & 2.6759335 \\
$2^{n+1}-1$ & 3.4823309 & 2.6807257 \\
$2^{n} n+1$ & 3.4827397 & 2.6815415 \\
$2^{n}(n+1)$ & 3.4824007 & 2.6812335 \\
$3^{n}-3^{n-1}+1$ & 3.4836193 & 2.6827835 \\
\hline
\end{tabular}

points and, in fact, coincides with the above Formula III. To our knowledge, however, Formula III has never been constructed by this technique. The advantage of using Lobatto formulas in constructing spherical product formulas was first pointed out to us by R. A. Sack; to our knowledge he was the first to propose this. The number of points in spherical product formulas of degree $2 m-1$, for general $m$, constructed from Lobatto formulas is given by Stroud and Secrest [8].

To illustrate the use of the formulas we have derived we numerically evaluate the integral

$$
J_{n} \equiv \int \underset{s_{n}}{\cdots} \int \cos \left(x_{1}+\cdots+x_{n}\right) d x_{1} \cdots d x_{n}
$$

where $S_{n}$ is the unit $n$-sphere $(n \geqq 2)$. The exact value of this integral is given by

$$
J_{n}=c_{00}\left[1-\frac{1}{k_{1}}+\frac{1}{k_{1} k_{2}}-\cdots+\frac{(-1)^{m}}{k_{1} k_{2} \cdots k_{m}}+\cdots\right] \text {, }
$$

where, as before, $c_{00}$ is the volume of the $n$-sphere and

$$
k_{\alpha}=\frac{4}{n} \alpha^{2}+2 \alpha, \quad \alpha=1,2, \cdots .
$$

The approximate values obtained from our four formulas, and also the formulas containing $2 n^{2}+1$ and $3^{n}-3^{n-1}+1$ points, are given in Table 1 for $n=4,8$. The exact values; correct to 8 decimals, are

$$
\begin{aligned}
& J_{4}=0.705668057 \frac{\pi^{2}}{2}=3.48233228 \\
& J_{8}=0.660697220 \frac{\pi^{4}}{24}=2.68157983 .
\end{aligned}
$$

It should be noted that in 4 dimensions certain coefficients in the $2 n^{2}+1$ point formula are zero so, in effect, that formula uses only ${ }^{\prime} 5$ points.

Hudson Laboratories

Columbia University

Dobbs Ferry, New York 10522

1. V. A. Ditkin, "On certain approximate formulas for the calculation of triple integrals," Dokl. Akad. Nauk SSSR, v. 62, 1948, pp. 445-447. (Russian) MR 10, 331.

2. F. R. GANTMAHER, The Theory of Matrices, GITTL, Moscow, 1953; 2nd ed., 1963; English transl., Vols. 1, 2, Chelsea, New York, 1959. MR 16, 438; MR 21 \#6372c. 
3. P. C. Hammer \& A. H. Stroud, "Numerical evaluation of multiple integrals II," Math. Tables Aids Comput., v. 12, 1958, pp. 272-280. MR 21 *970.

4. R. G. Hetherington, "Numerical integration over hypershells," Thesis, University of Wisconsin, Madison, Wis., 1961.

5. I. P. MrsovskiH, "Cubature formulas for evaluating integrals over a sphere," Dokl. Akad. Nauk SSSR, v. 147, 1962, pp. 552-555 (Russian) = Soviet Math. Dokl., v. 3, 1962, pp. 16701673. MR 26 *4480.

6. W. H. PeIrCe, "Numerical integration over the planar annulus," J. Soc. Indust. Appl. Math., v. 5, 1957, pp. 66-73. MR 19, 771 .

7.'W. H. PeIRce, "Numerical integration over the spherical shell," Math. Tables Aids Comput., v. 11, 1957, pp. 244-249. MR $20 * 430$.

8. A. H. STroud \& D. Secrest, Gaussian Quadrature Formulas, Prentice-Hall, Englewood Cliffs, N. J., 1966. (To appear.) 